\title{
Diagnóstico laboratorial de enteroparasitoses oportunistas, com ênfase nas microsporidioses humanas, em Goiânia-G0
}

\author{
Laboratory diagnosis of opportunistic intestinal parasites with \\ emphasis on human microsporidiosis, in Goiânia-GO
}

\author{
Edson Sidião de Souza Júnior ${ }^{1}$ e Marco Tulio A. Garcia-Zapata ${ }^{1}$
}

\begin{abstract}
RESUMO
Os microsporídios são protozoários, emergentes e oportunistas, responsáveis por patologias de alta morbi-mortalidade, principalmente em indivíduos com distúrbios imunes. Este estudo visa determinar o perfil clínico-laboratorial destes agentes. No total, foram avaliados 723 pacientes divididos em dois grupos: I) Indivíduos imunodeprimidos/imunossuprimidos; II) Indivíduos aparentemente imunocompetentes. Estes, após livre e esclarecido consentimento, foram entrevistados e cederam amostras fecais, sendo todas submetidas a técnicas de HPJ, Rugai, Faust e colorações específicas para coccídios e microsporídios. A freqüência de microsporídios foi 1,3\% (5/393) no grupo I, enquanto no outro grupo foi quatro vezes menor. A ocorrência de outras enteroparasitoses oportunistas também foi maior no grupo I. Conclui-se, por um lado, que estes agentes estão em nosso meio, e por outro, necessitamos aprimorar o diagnóstico clínico e laboratorial, para definir a distribuição geográfica destes agentes no Estado de Goiás e no Brasil.
\end{abstract}

Palavras-chaves: Microsporidioses. Enteroparasitas oportunistas. Diagnóstico laboratorial.

\begin{abstract}
Microsporidia are emergent and opportunistic protozoa that are responsible for diseases with high morbidity and mortality, especially among individuals with immune disorders. This study had the aim of determining the clinical-laboratory profile of these agents. In total, 723 patients were evaluated, divided into two groups: I) Immunosuppressed/immunodepressed individuals; II) Apparently immunocompetent individuals. After obtaining free informed consent, these patients were interviewed and gave fecal samples. These samples were all subjected to the HPJ, Rugai and Faust techniques and to specific staining for Coccidia and Microsporidia. The frequency of Microsporidia was 1.3\% (5/393) in group I, whereas it was a quarter of this in group II. The occurrence of other opportunistic intestinal parasites was also greater in group I. It was concluded, firstly, that these agents are present in our environment and, secondly, that there is a need to improve the clinical and laboratory diagnosis, in order to define the geographic distribution of these agents in the State of Goiás and throughout Brazil.
\end{abstract}

Key-words: Microsporidiosis. Opportunistic intestinal parasites. Laboratory diagnosis.

As infecções oportunistas são relatadas freqüentemente em pacientes imunodeprimidos, em especial naqueles com deficiência na atividade do sistema imunológico, causando um amplo espectro clínico-epidemiológico e laboratorial. Com o advento da pandemia da síndrome da imunodeficiência adquirida (SIDA), observamos o surgimento de algumas doenças consideradas como emergentes ou re-emergentes, sendo que a maioria dos agentes etiológicos envolvidos só tinha importância na medicina-veterinária ${ }^{29}$. Entre estes agentes emergentes/re-emergentes, encontramos os coccídios intestinais (Cryptosporidium parvum, Isospora belli e Cyclospora cayetanensis) e os microsporídios (Filo Microspora), protozoários entéricos oportunistas, responsáveis por uma diversidade de quadros clínicos com infecções refratárias ou incuráveis, com significativas causas de morte em pacientes HIV positivos e com Aids com contagem de linfócitos TCD4 inferior a 100-200 céls/mm $\mathrm{mm}^{132} 24$

\footnotetext{
1. Instituto de Patologia Tropical e Saúde Pública da Universidade Federal de Goiás, Goiânia, GO. Apoio financeiro: CNPq, CAPES.

Endereço para correspondência: Dr. Marco Tulio A. Garcia-Zapata. IPTSP/UFG. Rua Delenda Rezende de Melo, esq. com $1^{a}$ Avenida, Setor Universitário. Caixa Postal 131, 74605-050 Goiânia, GO. Tel: 5562 3209-6120, Fax: $55623521-1839$.

e-mail: zapata@iptsp.ufg.br; sidiao@hotmail.com

Recebido para publicação em 01/11/2005

Aceito em 4/12/2006
} 
Entre as enfermidades intestinais, destacam-se as microsporidioses intestinais como uma das principais causas parasitárias em indivíduos imunodeprimidos pelo HIV, constituindo-se desta maneira em importantes agravos secundários, resultando no agravamento do estado geral do paciente, particularmente em decorrência de quadros diarréicos de difícil controle ${ }^{3}$. Apesar de existirem mais de 143 gêneros e 1.200 espécies pertencentes ao filo Microspora, somente 6 gêneros estão envolvidos em processos infecciosos em humanos ${ }^{8}$. Dentre as espécies envolvidas, Enterocytozoon bieneusi é a mais freqüentemente encontrada, seguida de Encephalitozoon intestinalis, anteriormente classificada como Septata intestinalis ${ }^{18}$, sendo que as duas espécies são comuns no trato gastrointestinal, podendo ocasionar infecção disseminada, especialmente por esta última espécie, com comprometimento do trato urinário, fígado, seios paranasais, vias biliares, trato respiratório, peritôneo, musculatura estriada, rins e $\mathrm{SNC}^{23}$.

Apesar da maioria destes parasitas estar em evidência por causa da SIDA, não tem sido dada atenção, quer pelo desconhecimento por parte dos profissionais de saúde, quer pelo fato de existir poucos laboratórios devidamente preparados para o estudo e caracterização dos agentes etiológicos envolvidos, apesar da existência de técnicas modernas, eficientes e viáveis de coloração coprológica disponíveis por microscopia de luz ${ }^{15}$. Dentre os vários métodos de coloração coprológica, destacam-se 0 Chromotrope de Weber e suas modificações ${ }^{14192124}$ para a identificação dos esporos de microsporídios. A mensuração dos esporos é fundamental na sua diferenciação com outros microorganismos, sendo indispensável utilizar a ocular micrométrica no diagnóstico destes agentes.

Além do registro constante de enteroparasitas de caráter oportunista, nos pacientes imunodeprimidos, principalmente naqueles com SIDA, estes agentes podem acometer indivíduos imunocompetentes, porém com quadro clínico menos severo e com prognóstico favorável ${ }^{18}$.

Ainda assim, esses agentes continuam pouco conhecidos e/ou estudados em nosso meio, razão pela qual este trabalho procurou avaliar a importância dos mesmos e, registrar os primeiros casos de microsporidioses e ciclosporíase intestinal humana no Estado de Goiás, com finalidade de caracterizar e subsidiar preferencialmente o conhecimento acerca dos aspectos clínicos e laboratoriais dessas enfermidades e a sua implicação nos indivíduos por eles infectados.

\section{MATERIAL E MÉTODOS}

Área e população do estudo. 0 estudo laboratorial foi realizado entre novembro de 1999 e dezembro de 2003, pela Unidade de Protozoologia do IPTSP/UFG, no qual avaliou 723 pacientes e examinou-se 987 amostras com média de 1,4 amostras por paciente. Esta população foi dividida em dois grupos: o Grupo I formado por 393 pacientes (657 amostras com média de 1,7 amostras por paciente), portadores de diarréia e/ou algum tipo de comprometimento do sistema imunológico (considerados pacientes HIV positivos e com Aids, pacientes com neoplasias e em tratamento com drogas quimioterápicas e pacientes fazendo o uso de drogas imunossupressoras) procedentes dos hospitais-escola da UFG e unidades referenciais em assistência à Saúde de Goiás, no município de Goiânia; e o Grupo II, formado paralelamente e de forma aleatória, no qual foram examinadas amostras fecais únicas de 330 indivíduos da comunidade aparentemente imunocompetentes, provenientes de Unidades Básicas de Saúde do SUS/GO ou encaminhadas pelas mesmas para o IPTSP/UFG, por estas unidades.

Coleta e processamento das amostras. Todas as amostras fecais foram coletadas em recipientes plásticos individuais, identificadas e divididas no qual: (1) parte foi conservada estocada a $-24^{\circ} \mathrm{C}$ para posterior estudo biomolecular; (2) parte foi mantida in natura; (3) parte preservada em solução aquosa de formalina a 10\%; (4) parte estocada em solução de bicromato de potássio a 2,5\%, com objetivo de induzir a esporulação de oocistos de Cyclospora sp, e conseqüentemente a visualização de dois esporocistos característicos no seu interior, auxiliando desta forma 0 diagnóstico laboratorial diferencial do Cryptosporidium parvum $^{13}$.

Diagnóstico parasitológico. Todas as amostras foram submetidas às técnicas comuns de exame parasitológico de fezes (HPJ, Rugai, Faust). As amostras conservadas em formalina a $10 \%$, foram submetidas à concentração pela técnica de formalina-acetato de etila ${ }^{20}$, com a finalidade de aumentar o número de oocistos e esporos no material fecal, para posterior coloração específica. A coloração específica para o diagnóstico de coccídios foi a de Kinyoun à quente e para microsporídios intestinais foi a de Hot-ChromotropeKokoskin ${ }^{16}$. Os achados laboratoriais sugestivos de infecção por esporos de microsporídios foram encaminhados para London School of Hygiene and Tropical Medicine (LSHTMUK), na Inglaterra, para confirmação e controle de qualidade.

Análise morfométrica. Um importante recurso laboratorial utilizado foi a análise morfométrica ${ }^{11}$, principalmente em virtude das características morfotintoriais

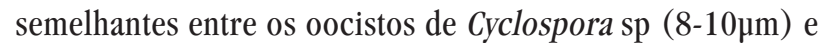
C. parvum (4-6 $\mu \mathrm{m})$. Este recurso também foi utilizado nas análises das partes de amostra a fresco e estocadas em solução de bicromato de potássio a 2,5\%.

Coleta e processamento dos dados. Para a coleta de dados foram elaboradas fichas padrão adequadas para a informatização e viabilização de um banco de dados. Estas foram aplicadas na análise dos prontuários médicos e na entrevista com os próprios pacientes.

Análise estatística. Os resultados obtidos foram repassados para um banco de dados, com posterior análise pelo Programa EPINFO versão 6.4d. A análise estatística foi realizada através do Teste $X^{2}$, com o objetivo de testar a significância dos resultados encontrados nos grupos estudados. 
Aspectos éticos. Os pacientes só foram incluídos no estudo, após eles próprios ou responsáveis, terem tomado conhecimento das informações básicas do mesmo (dos seus procedimentos e de seus riscos), e assinarem, na presença de uma testemunha, 0 "Termo de Consentimento Livre e Esclarecido". O presente estudo teve aprovação em Comitês de ética regionais (COEP-HDT/SESGO; COEP-HC/UFG; COEP-HGG).

\section{RESULTADOS}

Os resultados estão apresentados nas Tabelas 1 e 2 . Nos 723 pacientes estudados encontrou-se infecção por esporos sugestivos de microsporídios em 6 pacientes, representando aproximadamente 1\% do total estudado (Figura 1). Destes achados, 5 pacientes pertenciam ao grupo I e somente 1 ao grupo II.

Dos 393 pacientes avaliados do grupo I, 74 (19\%) pacientes estavam infectados por enteroparasitas, sendo que 18 (31\%) eram pacientes HIV positivos e com Aids e 3 (5,2\%)

Tabela 1 - Freqüência de pacientes encontrados com infecções por enteroparasitos patogênicos, no período de novembro de 1999 a dezembro de 2003-IPTSPIUFG.

\begin{tabular}{|c|c|c|c|c|c|c|}
\hline \multirow[b]{2}{*}{ Infecções } & \multicolumn{2}{|c|}{ Grupo I } & \multicolumn{2}{|c|}{ Grupo II } & \multicolumn{2}{|c|}{ Total } \\
\hline & $\mathrm{n}^{0}$ & $\%$ & $\mathrm{n}^{\mathrm{0}}$ & $\%$ & $\mathrm{n}^{0}$ & $\%$ \\
\hline Microsporidiose intestinal* & 5 & 1,3 & 1 & 0,3 & 6 & 1,0 \\
\hline Isosporíase* & 15 & 4,0 & 6 & 1,5 & 21 & 3,0 \\
\hline Criptosporidiose* & 11 & 3,0 & 2 & 0,6 & 13 & 2,0 \\
\hline Ciclosporíase & 5 & 1,0 & 2 & 0,6 & 7 & 0,9 \\
\hline Giardíase* & 14 & 3,5 & 37 & 11,0 & 51 & 7,0 \\
\hline Estrongiloidíase* & 9 & 2,5 & 1 & 0,3 & 10 & 1,1 \\
\hline Outras parasitoses** & 15 & 4,0 & 29 & 9,0 & 44 & 6,0 \\
\hline Negativo & 319 & 81 & 253 & 77,0 & 571 & 80,0 \\
\hline Total & 393 & 54,0 & 330 & 46,0 & 723 & 100,0 \\
\hline
\end{tabular}

$* P>X^{2} .05(1)=3,84^{* * *}$ Incluem nestas parasitoses infecções por: Ascaris lumbricoides, Ancilostomidios, Hymenolepis nana, Enterobius vermicularis, Endolimax nana, Entamoeba coli, Pentatrichomonas hominis, Iodamoeba butschilli.

Tabela 2 - Freqüência de pacientes encontrados com infecções por enteroparasitas patogênicos no Grupo I, no período de novembro de 1999 a dezembro de 2003 (IPTSP $\backslash$ UFG), divididos pelos fatores de imunodepressão e/ou imunossupressão.

\begin{tabular}{|c|c|c|c|c|}
\hline Infecções & $\begin{array}{c}\text { Pacientes } \\
\text { HIV +/ SIDA }\end{array}$ & $\begin{array}{c}\text { Pacientes com } \\
\text { neoplasias }\end{array}$ & $\begin{array}{c}\text { Pacientes usuários } \\
\text { de drogas } \\
\text { imunossupressoras }\end{array}$ & Total \\
\hline Microsporidiose intestinal & 4 & 1 & 0 & 5 \\
\hline Isosporíase & 15 & 0 & 0 & 15 \\
\hline Criptosporidiase & 11 & 0 & 0 & 11 \\
\hline Ciclosporíase & 5 & 0 & 0 & 5 \\
\hline Giardíase & 12 & 1 & 1 & 14 \\
\hline Estrongiloidíase & 9 & 0 & 0 & 9 \\
\hline Amebíase & 0 & 1 & 0 & 1 \\
\hline Blastocistose & 0 & 0 & 2 & 2 \\
\hline Outras parasitoses & 10 & 1 & 1 & 12 \\
\hline Negativos & 293 & 23 & 15 & 319 \\
\hline Total & 347 & 27 & 19 & 393 \\
\hline
\end{tabular}

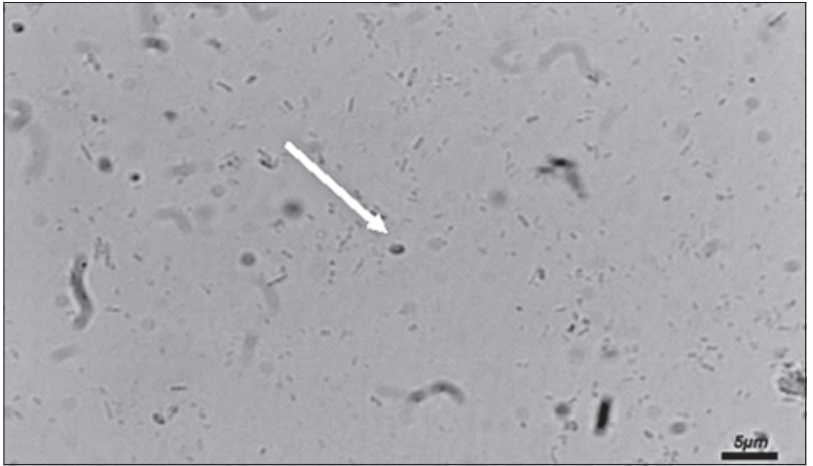

Figura 1 - Esporo de microsporídio corado pela técnica de HotChromotrope-Kokoskin (X1000), diagnosticado pelo IPTSP/UFG.

pacientes imunodeprimidos por outros fatores. Os microsporídios foram encontrados em $1 \%$ do total (5 pacientes), sendo a maioria com imunodeficiência pelo HIV e com Aids (4 pacientes). Destaca-se o encontro de um paciente com neoplasia infectado por microsporídios intestinais. Neste grupo, identificou-se, também: Isospora belli (4\%), Cryptosporidium sp (3\%) Giardia lamblia (3,5\%), Strongyloides stercoralis (2,5\%), Cyclosporasp (1\%) e outras parasitoses $(4 \%)$.

Nas amostras provenientes do Grupo II, foi encontrado somente um $(0,3 \%)$ paciente infectado por microsporídios intestinais. Constatou-se que em 77/330 (33\%) dos indivíduos de grupo apresentavam algum tipo de infecção por enteroparasita, nos quais se destacaram a Giardia lamblia (11\%), Isospora belli (1,5\%), Cryptosporidium sp (0,6\%), Cyclospora sp (2\%), Strongyloides stercoralis $(0,3 \%)$ e outras enteroparasitoses (9\%).

Observou-se diferença estatística pelo teste $X^{2}$ entre os grupos analisados, afirmando o caráter oportunista dos microsporídios intestinais que apresentaram maior freqüiência no grupo de pacientes com imunodepressão e/ou imunossupressão.

Dos 56 pacientes $\mathrm{HIV}^{+}$e com Aids para algum tipo de parasita oportunista, observou-se que 22 (40\%) apresentaram-se com diarréia aguda e 34 (60\%) com diarréia crônica. Em relação à contagem de linfócitos TCD4 $\backslash \mathrm{mm}^{3}$, $31(55 \%)$ dos pacientes estavam com contagem inferior a 50 cels $/ \mathrm{mm}^{3}, 13(23 \%)$ entre $50-100$ cels $/ \mathrm{mm}^{3}, 6$ (11\%) acima de 200 cels $/ \mathrm{mm}^{3}$ e 6 (11\%) cujo resultados não foram obtidos.

Duas amostras que tinham a presença de esporos sugestivos de microsporídios foram enviadas a LSHTM/ Londres-GB e confirmadas como sendo esporos de microsporídios através do uso de técnicas de coloração específicas e mensuração. Não foi possível a confirmação da espécie por microscopia eletrônica ou técnicas biomoleculares, dado a preservação das amostras em solução aquosa de formalina a $10 \%$, e este preservante interferir na reação de PCR ou processamento para análise por microscopia eletrônica. 


\section{DISCUSSÃo}

Com o surgimento da síndrome da imunodeficiência adquirida, observa-se a modificação dos padrões de ocorrência de muitas infecções nos pacientes acometidos inclusive, sendo necessária adequação da rotina parasitológica para o diagnóstico laboratorial desses agentes entéricos oportunistas e emergentes ${ }^{13}$.

Em nossa casuística, constatou-se que existe uma compatibilidade de frequiência de protozoários intestinais oportunistas nos pacientes do Grupo I e do Grupo II com outras casuísticas nacionais e mundiais ${ }^{3561016}$, mostrando que o uso de formas apropriadas de diagnóstico laboratorial por meios de técnicas de coloração coprológica está associado a caracterização destes agentes entéricos.

No referente aos microsporídios, foram detectados somente seis casos na nossa casuística, contrapondo desta maneira com dados da literatura especializada, que revelam uma incidência mais elevada nos pacientes com comprometimentos do sistema imunológico ${ }^{7812}$, em especial pelo HIV.

No Centro-Oeste brasileiro a ocorrência de microsporidioses parece ser muito baixa, pois em estudo prévio feito por nosso grupo, não foram demonstrados microsporídios em material coprológico ${ }^{10}$. Este fato pode estar relacionado à baixa prevalência, em nosso meio, de cepas pouco patogênicas e expressivas clinicamente, ou talvez, consequiência da terapia antiretroviral aplicada. Contudo, deve ser observado que dados que porventura venham discordar com os referendados na literatura médica especializada, podem ser consequiência da diversidade metodológica utilizada, tanto na seleção das amostras de estudo, como nos procedimentos laboratoriais utilizados.

Apenas em um paciente com imunodepressão e/ou imunossupressão teve o diagnóstico negativo na primeira amostra fecal analisada, tornando-se positivo para esporos de microsporídios na segunda. Os demais pacientes positivaram na análise da primeira amostra. Apesar das características biológicas próprias dos coccídios intestinais, particularmente no referente ao seu ciclo de vida, este fato assinala que os métodos de tinção coprológicos específicos para o diagnóstico parasitológico desses agentes são de fato eficientes, mesmo nos casos das análises parasitológicas em uma só amostra fecal.

No presente trabalho, os resultados mostraram que além da incidência das infecção por I. belli e Cryptosporidium sp nos pacientes do Grupo I ser superior às encontradas no Grupo II, os achados registraram os primeiros casos de infecção por microsporídios intestinais e Cyclospora sp. Foram também observadas freqüências mais elevadas de Strongyloides stercoralis entre os pacientes do Grupo I, quando comparados ao Grupo II.

É interessante ressaltar o encontro de oocistos de Cryptosporidium sp, I. belli e Cyclospora sp, que apesar de serem protozoários comuns em indivíduos imunodeprimidos, devido ao seu caráter oportunista, foram diagnosticados nos pacientes aparentemente imunocompetentes.
No total das amostras analisadas, chama atenção a baixa frequência de infecções por Entamoeba histolytica/dispar e por Blastocystis hominis, sendo estes parasitas relativamente comuns em nosso meio, relatados constantemente na literatura médica ${ }^{10}$. Tal fato, provavelmente, se deve a dificuldade em obter amostras recém coletada durante 0 estudo.

Em contraste, outras helmintíases patogênicas ao homem não foram identificadas entre os pacientes oriundos do Grupo I, contrapondo com as frequiências obtidas nos indivíduos atendidos nas unidades de atenção primária do município de Goiânia, pertencentes ao Grupo II. Acreditamos que esta diferença seja devido a poliquimioterapia que são submetidos os pacientes do Grupo I, mascarando os resultados laboratoriais.

Os resultados do presente trabalho mostram ainda, que os casos positivos para enteroparasitos oportunistas entre os pacientes imunodeprimidos pelo HIV, estão associados na sua maioria com quadros de diarréia e com contagem de linfócitos de CD4 inferior a 50-100 cels $/ \mathrm{mm}^{3}$, o que vem caracterizar o caráter oportunista dessas entidades.

Em relação aos achados encontrados nas amostras examinadas dos pacientes HIV negativos e imunodeprimidos por outros fatores, notamos que os resultados, na sua maioria, não comungam com aqueles obtidos entre os imunocomprometidos pelo HIV, talvez devido ao fato que somente os pacientes cujos resultados foram positivos para microsporídios encontravam-se com processo diarréico.

Contudo, considerando o encontro de esporos sugestivos de microsporídios intestinais em indivíduos não imunodeprimidos pelo HIV ou outro fator potencial de imunodepressão ou imunossupressão, é sentida a necessidade de avaliação destes parasitas em qualquer paciente que apresente quadros clínico diarréicos, independentes da sua resposta imune.

Nesse mesmo contexto, achamos conveniente recomendar a incorporação das técnicas de coloração coprológica específicas para o diagnóstico de microsporídios intestinais na rotina laboratorial, assim como o estudo morfométrico, quer nas unidades de referência em tratamento de pacientes $\mathrm{HIV}^{+}$e com Aids, quer em qualquer unidade e/ou laboratório que realize exames parasitológicos.

Por fim, deve ser considerada a implantação de programas educativos e preventivos, junto aos médicos, a outros profissionais da área de saúde e à comunidade em geral, visando ao controle desses parasitas emergentes e reemergentes, especialmente nos portadores do HIV, seguindo as recomendações da Organização Mundial de Saúde ${ }^{18}$. Além disso, deve ser considerado o desenvolvimento de estudos bio-moleculares em nossa região, para que possam distinguir a espécie de microsporídio que seja a responsável pela infecção. Essa distinção da espécie é fundamental na escolha do manejo terapêutico a ser adotado e na definição prognóstico da infecção. 


\section{REFERÊNCIAS BIBLIOGRÁFICAS}

1. Bornay-Llinares FJ, Silva AJ, Moura H, Schwartz DA, Visvesvara GS, Pieniazek NJ, Cruz-Lopez A, Hernandez-Jauregui P, Guerrero J, Enriquez FJ. Immunologic, microscopic and molecular evidence of Encephalitozoon intestinalis (Septata intestinalis) infection in mammals other than humans. Journal of Infectious Diseases 178: 820-826, 1998

2. Carneiro JR, Guimarães OS, Cury FC, Rodrigues N, Lima JD. Diagnóstico sorológico da Criptosporidiose humana. Revista de Patologia Tropical 24:205-217, 1995

3. Cimerman S, Cimerman B, Lewi DS. Enteric parasites and AIDS. São Paulo Medical Journal 117: 266-273, 1999.

4. Cimerman S, Cimerman B, Lewi DS. Prevalence of intestinal parasitic infections in patients with acquired immunodeficiency syndrome in Brazil. International Journal of Infectious Diseases 3:203-206, 1999.

5. Clavero AO, Verdu ME, Peman J, Dario R, Gobernado M. Human intestinal infection due to coccidia in Mozambique: two cases. Acta Tropica $72: 25-29,1999$

6. Cotte L, Rabodonirina M, Piens MA, Perreard M, Mojon M, Trepo C. Prevalence of intestinal protozoans in French patients infected with HIV. Journal Acquired Immune Deficiency Syndromes 6:1024-1029, 1993.

7. Fedorko DP, Hijazi YM. Application of molecular techniques to the diagnosis of Microsporidial infection. Emerging Infectious Diseases 2: 183-191, 1996.

8. Field AS, Canning EU, Hing MC, Verre J, Marriott D. Microsporidia in HIVinfected patients in Sidney, Australia: a report of 37 cases, a new diagnostic techinique and the light microscopy and ultrastructure of a disseminated species. AIDS 7: S27-S33, 1993.

9. Franzen C, Müller A. Molecular Techniques for Detection, Species Differentiation, and Phylogenetic Analysis of Microsporidia. Clinical Microbiology Reviews 12:243-285, 1999.

10. Garcia-Zapata MTA, Cuba CC, Silva AE, Leite SA, Reis MI, Moreira AF, Maltarollo TAP, Gomes R, Fonseca RA. Infecções por Parasitas Oportunistas em Pacientes HIV \SIDA Positivos, no Hospital Universitário de Brasília. Brasília Médica 37:14-18, 2000.

11. Gonzáles-Ruiz A, Bendall R. Size matters: the use of the ocular micrometer in diagnostic parasitology. Parasitology Today 11:83-85, 1985

12. Gool VT, Luderhoff E, Nathoo KJ, Kire CF, Dankert J, Mason PR. High prevalence of Enterocytozoon bieneusi infections among HIV-positive individuals with persistent diarrhoea in Harare, Zimbabwe. Transactions of the Royal Society of Tropical Medicine and Hygiene 89:478-480, 1995.

13. Hamour AA, Mandal BK. Coccidian Parasites in patientes with AIDS: Cryptosporidiosis, Microsporidiosis, Isosporiasis and Cyclosporiasis. Balliere's Clinical Infectious Diseases 3:137-153,1996.

14. Harstskeerl RA, Van Gool T, Schuitema AR, Didier ES, Terpstra W. Genetics and immunological characterization of the microsporidial Septata intestinalis Cali, Kotler and Orestion, 1993: reclassification to Encephalitozoon intestinalis. Parasitology 110: 277-285, 1995.

15. Kokoskin E, Gyorkos TW, Camus A, Cedilotte L, Purtill T, Ward B. Modified technique for efficient detection of microsporidia. Journal Clinical of Microbio1ogy 32:1974-1975,1994

16. Lainson R, Silva BAM. Intestinal parasites of some diarrhoeic HIVseropositive individuals in North Brazil, with particular reference to Isospora belli Wenyon, 1923 and Dientamoeba fragilis Jepps \& Dobell, 1918. Memórias do Instituto Oswaldo Cruz 94:611-613, 1999.

17. Mohandas K, Sehgal R, Sud A, Malla N. Prevalence of Intestinal Parasitic Pathogens in HIV-Seropositive Individuals in Northern India. Japanese Journal of Infectious Diseases 55: 83-84, 2002.

18. Organização Panamericana de Saúda. Pautas para la prevención de infecciones oportunistas en personas con VIH/SIDA en América Latina y el Caribe. $3^{\text {a }}$ edition. Washington, DC, 2000.

19. Pape JW, Rose-Irene V, Boncy M, Boncy J, Johnson WD. Cyclospora Infection in Adults Infect with HIV. Annals of Internal Medicine 121:654657,1994 .

20. Ridley DS, Hawgood BC. The value of formol-ether concentration of faecal cysts and ova. Journal Clinical of Pathology 9:74-76, 1956.

21. Sauda FC, Zamarioli LA, Ebner Filho E. Prevalence of Cryptosporidium sp. and Isospora belli amoung AIDS patients attending Santos Reference Center for AIDS, São Paulo, Brazil. Journal of Parasitology 79:454-456, 1993.

22. Sauda FC, Zamarioli LA, Ebner Filho W, Mello LB. Prevalence of Cryptosporidium sp. and Isospora belli among AIDS patients attending Santos Reference Center for AIDS, Sao Paulo, Brazil. Journal of Parasitology 79:454-456, 1993

23. Sterling CR e Ortega YR. Cyclospora: an Enigma Worth Unraveling. Emerging Infectious Diseases 5:48-53, 1999.

24. Weber R, Bryan RT, Schwartz DA, Owen RL. Human Microsporidial Infections. Clinical Microbiology Reviews 7: 426-461, 1994. 